\title{
The treatment of chronic pleural empyema with laparoscopic omentoplasty. Initial report
}

\author{
Wojciech Żurek ${ }^{1}$, Wojciech Makarewicz ${ }^{2}$, Maciej Bobowicz ${ }^{2}$, Wioletta Sawicka ${ }^{3}$, Witold Rzyman ${ }^{1}$ \\ ${ }^{1}$ Department of Thoracic Surgery, Medical University of Gdansk, Gdansk, Poland \\ ${ }^{2}$ Department of Surgical Oncology, Medical University of Gdansk, Gdansk, Poland \\ ${ }^{3}$ Department of Anaesthesiology and Intensive Care, Medical University of Gdansk, Gdansk, Poland
}

Videosurgery Miniinv 2014; 9 (4): 548-553

DOI: $10.5114 /$ wiitm.2014.45129

\begin{abstract}
Introduction: Pleural empyema is the most serious, life-threatening postoperative complication of pneumonectomy, observed after 1-12\% of all pneumonectomies, with bronchopleural fistula being its main cause.

Aim: The aim of this publication is to present early outcomes of minimally invasive surgical management of pleural empyema. Patients were subjected to a single, complex procedure, consisting of the laparoscopic mobilization of the greater omentum and its transposition via the diaphragm into the pleural cavity to fill in the empyema cavity with the consecutive pleuro-cutaneous fistuloplasty (thoracoplasty).

Material and methods: Between May 2011 and April 2013, 8 patients were qualified to undergo the procedure. The mean age was 61 years (range: 46-77 years). Presence of bronchopleural fistula was confirmed in 3 cases. The median time of treatment with thoracostomy was 14.5 months.

Results: The mean operative time was $125 \mathrm{~min}$. The mean duration of post-operative hospital stay was 13.5 days (range: 7-31 days). In 6 patients (75\%) the objective of permanent resolution of pleural empyema was achieved. In total, 4 patients had complications: pleural empyema recurrence (2 patients), splenic injury, hiatal hernia, gastrointestinal bleed. Two patients with empyema recurrence had Staphylococcus aureus infections prior to surgery. They were successfully managed both with prolonged thoracic drainage and antibiotics.

Conclusions: Use of the greater omentum that was laparoscopically mobilized and transpositioned into the pleural cavity allows simultaneous management of the pleural empyema cavity and thoracostomy. The procedure is safe, with few direct complications. It is well tolerated and has at least a satisfactory cosmetic effect. The minimally invasive approach allows faster recovery and return to daily activities in comparison to the fully open technique.
\end{abstract}

Key words: pleural empyema, bronchopleural fistula, minimally invasive surgery, laparoscopic omentoplasty, pleuro-cutaneous fistuloplasty, thoracoplasty.

\section{Introduction}

Thoracic procedures carry a high burden of complications, including infectious complications. Pleural empyema is the most serious and life-threatening postoperative complication of pneumonectomy, with a rate of $1 \%$ to $12 \%$ [1-3]. In over $80 \%$ of cases, bronchopleural fistula is the main cause of pleural empyema [4]. Surgical management of pleural empyema is a long-term and multistep treatment. It consists of pharmacological treatment with antibiotics, closed drainage of the pleural cavity, creation of a thoracostomy, and finally when the pleural cavity is cleaned, it is filled with a transpositioned muscular

\section{Address for correspondence}

Wojciech Żurek MD, PhD, Department of Thoracic Surgery, Medical University of Gdansk, 7 Dębinki St, 80-299 Gdansk, Poland, phone: +48 502267 106, e-mail: zurek1@gumed.edu.pl 
flap or greater omentum with simultaneous reconstruction of the skin closure. This approach ensures at least an $80 \%$ success rate in terms of closure of the defect and the cosmesis [5]. Most importantly, it allows the patient to return to normal activities. It is successful under the condition that there is no bronchopleural fistula or the fistula was sealed in a way that would ensure its closure in a short period of time. Patients' own tissues, e.g. a pedicled muscular flap or the greater omentum transpositioned into the pleural cavity, provide the greatest chance for fistula closure. Surgical mobilization of the mus cular flap or harvesting the greater omentum via laparotomy additionally strains the patient and delays recovery. Minimally invasive laparoscopically assisted harvesting of the greater omentum and its transposition into the pleural cavity significantly reduces perioperative stress and allows faster postoperative convalescence.

\section{Aim}

The aim of this publication is to present early outcomes of minimally invasive surgical management of pleural empyema in 8 patients. The patients were subjected to a single complex procedure consisting of the laparoscopic mobilization of the greater omentum and its transposition via the diaphragm into the pleural cavity to fill in the empyema cavity with the consecutive pleuro-cutaneous fistuloplasty (thoracostomy).

\section{Material and methods}

\section{Patients' characteristics}

Between May 2011 and April 2013, eight patients were qualified to undergo elective pleuro-cutaneous fistula closure with reconstructive laparoscopic mobilization and transposition of the greater omentum into the pleural cavity. The mean age was 61 years (range: 46-77 years). Presence of bronchopleural fistula was confirmed in 3 cases. The number and extent of the primary procedure are shown in Table I.

In half of all patients the indication for the primary procedure was lung cancer (Table II).

The median time of treatment with thoracostomy was 14.5 months (range: 5-37 months). Prior to the procedure, the treatment plan was discussed in detail with every patient including treatment steps, expected final outcome and possible complications. All patients signed informed consent.
All patients were admitted to hospital seven days before the planned surgery. During that time, mechanical cleaning of the pleural cavity was performed, pleural smears were taken and microbiology assessment was sought. Microbiology reports were collated in Table III. In cases where pathogens were identified, the number of pathogens was described as 'single colonies only'. Macroscopic evaluation of the pleural cavity in all cases defined it as 'clean' (Table III).

\section{Surgical technique}

All operations were performed according to a standard two-step procedure. Each procedure started with the abdominal stage of laparoscopic mobilization of the greater omentum. The patient was placed in the supine position. The first, optical

Table I. Number and extent of primary procedures $(n=8)$

\begin{tabular}{|lcc|}
\hline $\begin{array}{l}\text { Extent of } \\
\text { pneumonectomy }\end{array}$ & \multicolumn{2}{c|}{$\begin{array}{c}\text { Site of primary procedure } \\
\text { and number of procedures }\end{array}$} \\
\cline { 2 - 3 } & Right & Left \\
\hline Pulmonectomy & 4 & 2 \\
\hline Upper bilobectomy & 1 & 0 \\
\hline Wedge resection & 0 & 1 \\
\hline
\end{tabular}

Table II. Indication for primary procedure $(n=8)$

\begin{tabular}{|lc|}
\hline Diagnosis & Number of patients \\
\hline Non-small cell lung cancer & 4 \\
\hline Pleural empyema & 2 \\
\hline Pulmonary tuberculosis & 1 \\
\hline Fibrotic/cirrhotic lung changes & 1 \\
\hline
\end{tabular}

Table III. Results of pleural smears

\begin{tabular}{|lc|}
\hline Pathogen & Number of patients $(n=8)$ \\
\hline None & 3 \\
\hline Klebsiella pneumoniae & 1 \\
\hline Serratia marcescens & 1 \\
\hline Pseudomonas aeruginosa & 1 \\
\hline Staphylococcus aureus & 1 \\
\hline Staphylococcus aureus (MRSA) & 1 \\
\hline
\end{tabular}




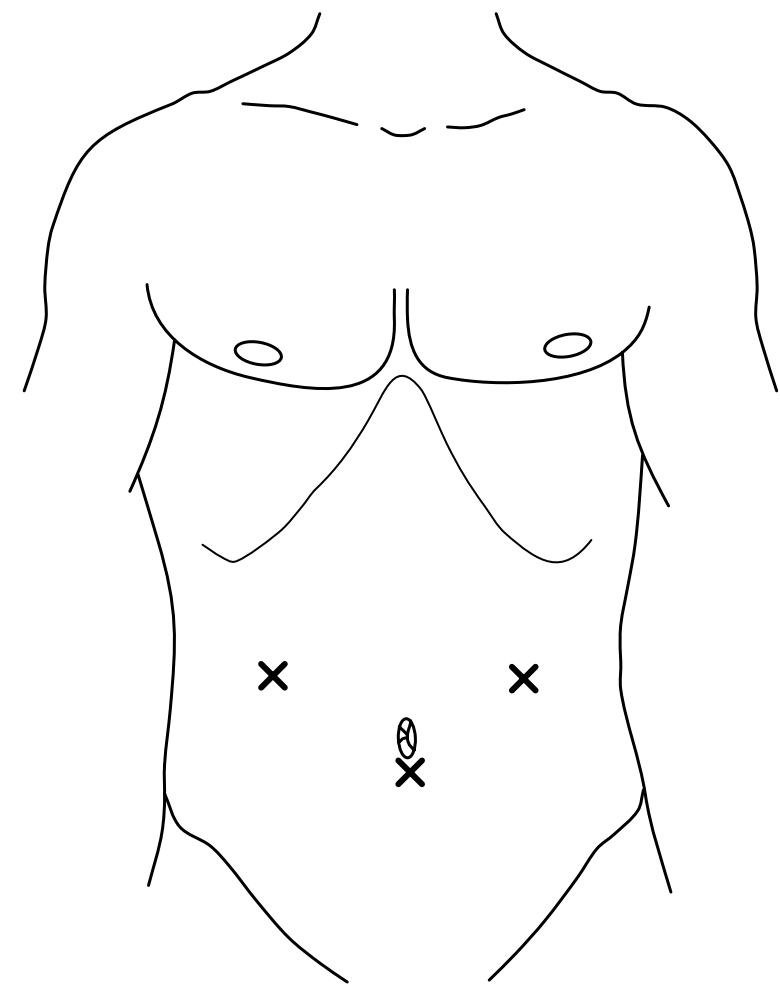

Figure 1. Location of ports

port $(11 \mathrm{~mm})$ was placed in the periumbilical area with open Hasson technique (Figure 1, Photo 1).

Then the $\mathrm{CO}_{2}$ was insufflated until the pressure of $10-12 \mathrm{~mm} \mathrm{Hg}$ was achieved. Next two trocars $(5.5 \mathrm{~mm})$ were placed in the right and left epigastrium. In two cases an additional $5.5 \mathrm{~mm}$ port was required due to the technical difficulties. Five-millimeter grasper and bipolar LigaSure (Covidien, USA) vessel sealing instruments were used for greater omentum mobilization. After visualization of the greater omentum and dissection of abdominal adhesions, the proper omental harvesting was performed. The gastric wall was elevated using a grasper, then

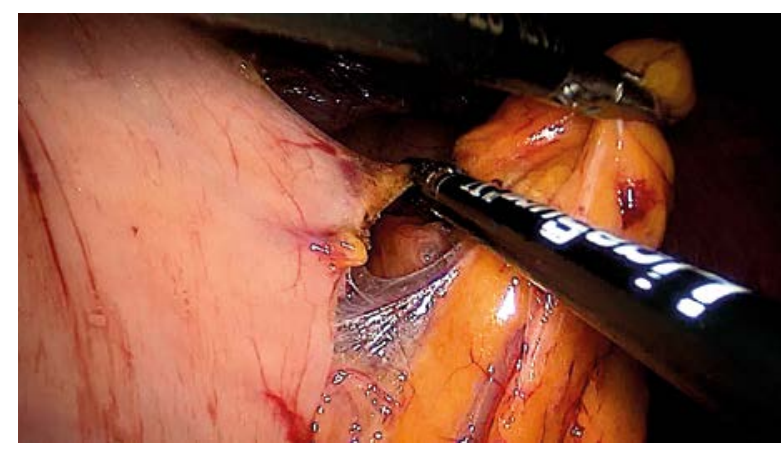

Photo 2. Dissection of greater omentum from gastric wall

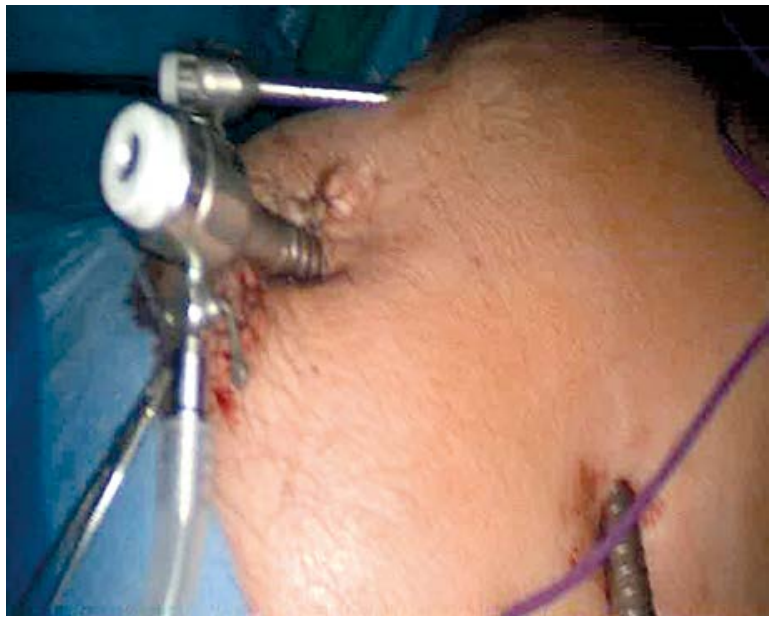

Photo 1. Location of ports

the right gastro-epiploic artery was visualized and isolated. Next, all the gastro-epiploic artery branches from the right to left side along the greater curvature of the stomach were sealed and dissected with the use of LigaSure (Photo 2).

When the left gastro-epiploic artery was visualized, the omentum was freed from the transverse colon, ensuring proper vascularization of both structures. When the omentum was dissected from the greater curvature of the stomach and from the transverse colon, the left gastro-epiploic artery was sealed with polymer Hem-O-Lock (Teleflex) clips and dissected with LigaSure (Photo 3).

The right gastro-epiploic vessels remained the principal vascularization for the harvested omentum. We did not use the omental dissection technique to extend the omentum. Following such preparation, the omental flap was placed in the epigastrium and the thoracic stage of the procedure was performed.

The thoracic part of the procedure started with mobilization of the skin edges sewn into the tho-

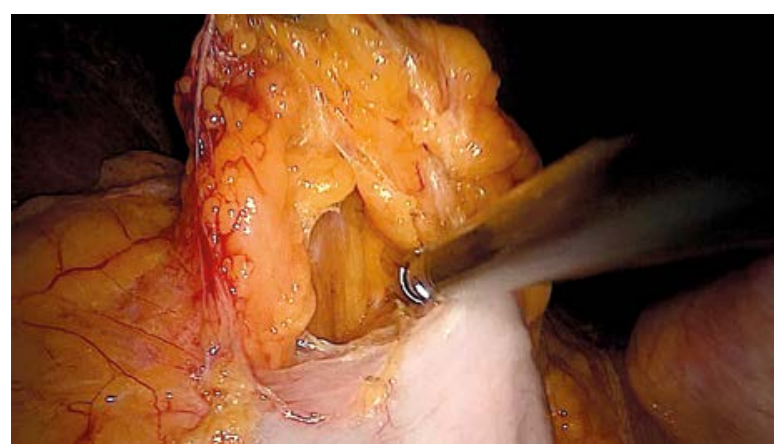

Photo 3. Dissection of greater omentum from transverse colon 


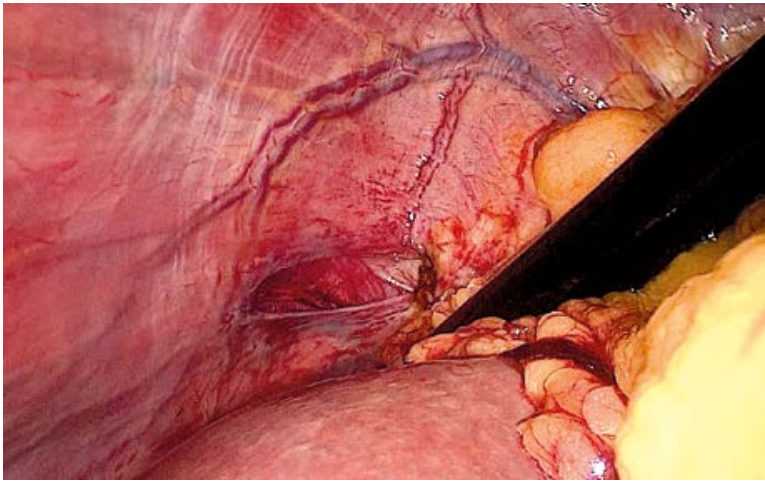

Photo 4. Opening in the diaphragm

racostomy opening. In some cases it was necessary to shorten the rib stumps that formed the border of the thoracostomy aperture. Next, the diaphragm was highlighted from the abdomen using laparoscopic optics to visualize its vascularization and phrenic nerve. Under direct vision, through the thorax, the diaphragm was incised radially for $4-5 \mathrm{~cm}$ up to the tendinous part of the diaphragm, ensuring that all vessels and the phrenic nerve were secured (Photo 4).

The next stage was translocation of the harvested omentum from the abdomen to the thoracic cavity. This was a crucial step that required gentle handling of the omentum. It was handed over using a grasper from the abdomen via the diaphragmatic opening to the post-resective pleural cavity (Photo 5).

The size of the diaphragmatic opening was controlled when the omentum was already transpositioned. Omental blood supply was checked for any impairment (Photo 6).

Then the omentum was fixed to the diaphragm with a few sutures.

This ensured that the omentum would not be retracted back to the abdominal cavity. The omentum was fixed with a few more sutures to the apex of the post-resective pleural cavity and the rest of it was placed freely in the cavity to equally fill up its volume. Part of the omentum was sewn over the bronchopleural fistula in cases of its presence. The intention of this was to reinforce the bronchial stump and to seal the fistula. In all cases we placed thoracic drains. At the end of the procedure we restored the skin continuity over the thoracostomy.

\section{Results}

The mean operative time was 125 min (range: 90-185 min). The mean duration of post-operative

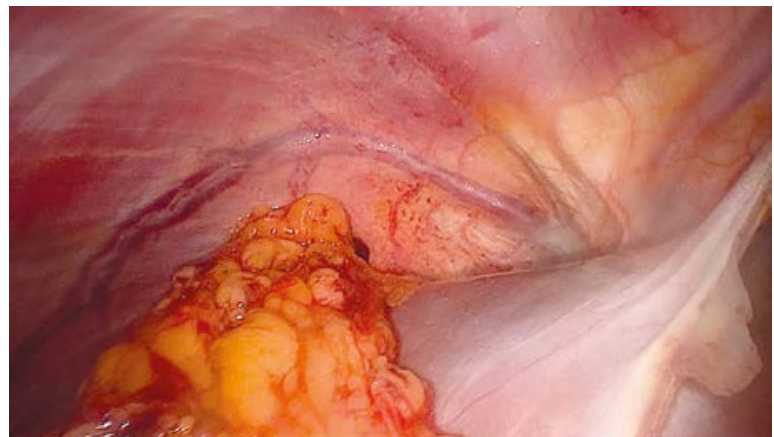

Photo 5. Translocation of harvested omentum to the thoracic cavity

hospital stay was 13.5 days (range: 7-31 days). In 6 patients (75\%) the objective of permanent resolution of pleural empyema was achieved. In total, 4 patients had complications: 3 had an early and 1 had a late complication (4 months after surgery). Characteristics of all complications are shown in Table IV.

An attempt of freeing omental adhesions near the splenic pole resulted in 1 patient having a traumatic injury to the spleen. It was managed through the conversion to an open procedure and application of the TachoSil fibrin sealant patch.

In another case, the gastrointestinal bleeding was the first manifestation of comorbid early gastric cancer. In the course of hospitalization the patient underwent further diagnostics for the gastric cancer und underwent gastrectomy at a later date.

We managed to close the skin over the thoracostomy site in all patients. The patients' satisfaction level with the procedure was at least good, including the 2 patients with pleural empyema recurrence. There were no perioperative deaths.

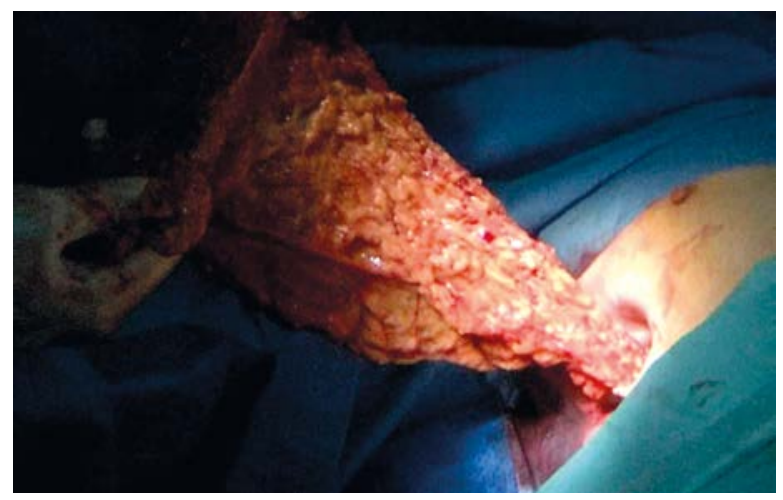

Photo 6. Omentum transpositioned through thoracostomy 
Table IV. Number and type of complications

\begin{tabular}{|lcc|}
\hline Type of complication & \multicolumn{2}{c|}{ Number of cases } \\
\cline { 2 - 3 } & Early & Late \\
\hline Pleural empyema recurrence & 1 & 1 \\
\hline Splenic injury & 1 & $(-)$ \\
\hline Hiatal hernia & 1 & $(-)$ \\
\hline Gastrointestinal bleeding & 1 & $(-)$ \\
\hline
\end{tabular}

\section{Discussion}

We are aware that 8 patients constitute a rather small cohort. Nevertheless, the innovative minimally invasive character of the procedure that combines laparoscopic mobilization of the greater omentum and subsequent thoracoplasty justifies presentation of early results. Combined thoraco-abdominal access makes an impression of rather extensive surgery and thus a serious burden for patients. Interestingly, in opposition to the above, patients described the postoperative pain sensation as rather mild and connected only with the thoracic wound. It can be easily explained as an effect of the minimally invasive laparoscopic access to the abdominal cavity.

The whole procedure is preceded by extensive preparation. The early stage of preparation is mechanical cleaning of the pleural empyema cavity to evacuate debris and pus via frequent change of the sterile gauze. This stage takes several months. Periodic microbiological monitoring in the outpatient department allows monitoring of the cleanliness of the empyema cavity. Negative smear test results were a prerequisite condition to qualify for omentoplasty. That is why all patients were admitted a week before planned surgery. In all cases microbiological cultures were done on admission and adequate steps were taken to ensure eradication of any pathogens from the empyema cavity. Major qualification criteria for omentoplasty are the lack of pus and the presence of healthy granulomatous tissue in the empyema cavity [6]. Presence of single pathogen colonies in the last smear test was noted in a few cases but due to the phagocytic properties of the greater omentum the risk seemed to be very low and thus acceptable.

Due to the complex character of the described procedure the complication burden remains high. Complications analysis showed that 5 complications occurred in 4 patients. Two patients with empyema recurrence had Staphylococcus aureus infections prior to surgery. They were successfully managed both with thoracic drainage and antibiotics. We believe that microbiological cleanliness is desirable but not obligatory for the surgical management of pleural empyema when macroscopic cleanliness is achieved. This idea has been supported not only by our observations but also by published experience of other authors, who stress that the greater omentum has huge phagocytic capabilities that ensure full control over the infection site [7]. On the other hand, preoperative targeted antibiotic therapy is recommended in all cases of bacterial colonization of the empyema cavity.

Two complications resulted directly from the operative technique. One was splenic injury and bleeding in a patient who had prior abdominal surgery with adhesions. The other was diaphragmatic hernia. A case of gastrointestinal bleeding due to undiagnosed gastric cancer might or might not be considered a complication.

Finally, the failure rate of $25 \%$ in terms of early or late recurrence of pleural empyema is comparable to open procedures [6].

The presented study shows that laparoscopically assisted omentoplasty is a safe procedure with acceptable complication and failure rates. Most complications were not directly connected with the type of used technique but rather with a complicated clinical setting of each patient.

\section{Conclusions}

Use of the greater omentum that was laparoscopically mobilized and transpositioned into the pleural cavity allows simultaneous management of the pleural empyema cavity and thoracostomy. The procedure is safe with few direct complications. It is well tolerated and has at least a satisfactory cosmetic effect. The minimally invasive approach allows faster recovery and return to daily activities in comparison to the fully open technique.

\section{References}

1. Taghavi S, Marta GM, Lang G, et al. Bronchial stump coverage with a pedicled pericardial flap: an effective method for prevention of postpneumonectomy bronchopleural fistula. Ann Thorac Surg 2005; 79: 284-8.

2. Regnard JF, Alifano M, Puyo P, et al. Open window thoracostomy followed by intrathoracic flap transposition in the treatment of empyema complicating pulmonary resection. J Thorac Cardiovasc Surg 2000; 120: 270-5. 
3. Forrester-Wood CP. Bronchopleural fistula following pneumonectomy for carcinoma of the bronchus. Mechanical stapling versus hand suturing. J Thorac Cardiovasc Surg 1980; 80: 406-9.

4. Kacprzak G, Marciniak M, Addae-Boateng E, et al. Causes and management of postpneumonectomy empyemas: our experience. Eur J Cardiothorac Surg 2004; 26: 498-502.

5. Chichevatov D, Gorshenev A. Omentoplasty in treatment of early bronchopleural fistulas after pneumonectomy. Asian Cardiovasc Thorac Ann 2005; 13: 211-6.

6. Botianu AM, Botianu PV. Modified thoraco-mediastinal plication (Andrews thoracoplasty) for post-pneumonectomy empyema: experience with 30 consecutive cases. Interact Cardiovasc Thorac Surg 2013; 16: 173-8.

7. Kitano M. Omentoplasty in thoracic surgery. Gen Thorac Cardiovasc Surg 2008; 56: 483-9.

Received: 13.07.2014, accepted: 15.07.2014. 